$\xi=-1$

\title{
A Review on Generation Management in India with Renewable Energy Sources
}

\author{
R.Rajesh $^{1 *}$, A.Mutharasan ${ }^{2}$ \\ ${ }^{1}$ Vel Tech Rangarajan Dr. Sagunthala R\&D Institute of Science \& Technology, Chennai \\ ${ }^{2}$ Vel Tech High Tech Dr. Rangarajan Dr. Sakunthala Engineering College, Chennai \\ *Corresponding Author E-mail: ${ }^{1}$ rajeshpse15@gmail.com
}

\begin{abstract}
Regarding development in the request and utilization of vitality. India is developing exponentially. With the request of spotless and environmentally friendly power vitality, it is important to tackle the tremendous potential from the sustainable power source part. With the GOI strategy and rules, around nine level of the aggregate introduced limit originates from the inexhaustible sources. With the expanded to the framework and observed to keep up the recurrence and members in the age administration framework. Given the vulnerability and inconstancy in the yield of generator, it is a noteworthy test to keep up the matrix with this vacillation. Under this viewpoint, this paper underscores the technique to address the difficulties in incorporating the power from sustainable power sources. Quantum of wind vitality is corresponded with the breeze speed and heading, shrewd estimate motor is embraced to lessen the uncertainly.
\end{abstract}

Keywords: Renewable Energy, Wind Power, Wind Forecast, Predictive Analysis \& Proactive decision.

\section{Introduction}

The demand for power is India is witnessing an exponential growth. The availability of energy is considered as one of the catalyst for economic growth. The envisaged growth cannot be achieved without meeting the energy requirement in a sustainable manner. The huge difference in the per capita consumption of electricity in the India (around $108.167 \mathrm{kWh} /$ annum) [1] compared to the world average (33.23\% of the world average) [2], signifies a tremendous growth potential in the field of installed capacity of electricity generation.

With average 9.9\% [1] local growth, more than $6.8 \%$ [1] shortage during the peak hours, demand around 169130 MW capacity additions in the next 10 years [1]. Growing concerns about global warming, demand for clean energy, climate change policies worldwide are the major drivers of global renewable energy development.

The Government of India is encouraging domestic and foreign investors to establish renewable energy based power generation projects by providing exemptions/ reductions in the tax, incentives and a definite power purchase agreement [1].

\section{Growth of Renewable Energy in India}

India is among the top 3 countries in the world with regard to installed power generation capacity from renewable energy sources [1]. Cumulative achievements of power from renewable sources till March 2018 is 34,046 [1] (which is around 9\% [1] of the total installed capacity. Wind energy has the posted the highest growth (180601 MW) [1].

In order to promote renewable energy, Electricity Regulatory Commission has mandated renewable purchase obligations.
Discoms are required to source up to $10 \%$ [1] of their power from RE sources. Different favorable government policies, generation based incentives (GBI), tax holidays will accelerate the growth and investment in the renewable energy sector.

\section{Potential of Renewable Energy in India}

The major renewable energy sources in India are wind, Hydro, Biomass and Solar. Having tremendous potential to harness. India is considered one of the ideal investment destinations for renewable generation.

India has an estimated medium term renewable energy potential more than 1,542,650 MW [1],[2],[3] from commercially exploitable sources. Of them wind power potential holds the major share around 1,186,653 MW[1],[2],[3], followed by energy from biomass with a potential around 15,252 MW [2], [3]. In addition, the potential to generate power from solar energy is $25,871,000$ MW [2].

Even at the conservative assessment, total estimated potential of 25,643,027 MW (without considering the solar energy sector), the investment potential in the country is Rs. 11.5 lakh Crore 8 [1].

The Greenpeace International, European Renewable Energy (EREC) in its report released in March, 2009 has projected that by 2050 ; about $69 \%$ of the electricity produced in India will come from renewable energy sources [1].

\section{Monitoring of Renewable Generation}

As of now not very many of the sustainable generators are associated with the lattice and are observed. Age from sustainable sources will expand step by step. Also, it is the need of hour to associate them with the matrix to direct recurrence. This won't just 
decrease the weight of the ordinary vitality sources, particularly the petroleum derivatives, yet in addition enhance the general age cost and lessen the general carbon impression.

\section{Challenges in Integration of Renewable Generation}

Preceding incorporating sustainable power source, three viewpoints - Physical, Operational and Informational mix must be investigated. Yields from these generators are for the most part factor and indeterminate.

- Variability: The yield of generators changes as per the accessibility of the essential fuel ( wind, daylight) bringing about expanded vacillations in the plant yield on unsurpassed scale.

- Uncertainty: The capacity to figure the greatness and phase (i.e. timing) of generator yield is less unsurprising than for regular age. Wind or sun oriented power isn't generally there when it is required most. The figure beneath clarifies its uncorrelated conduct.

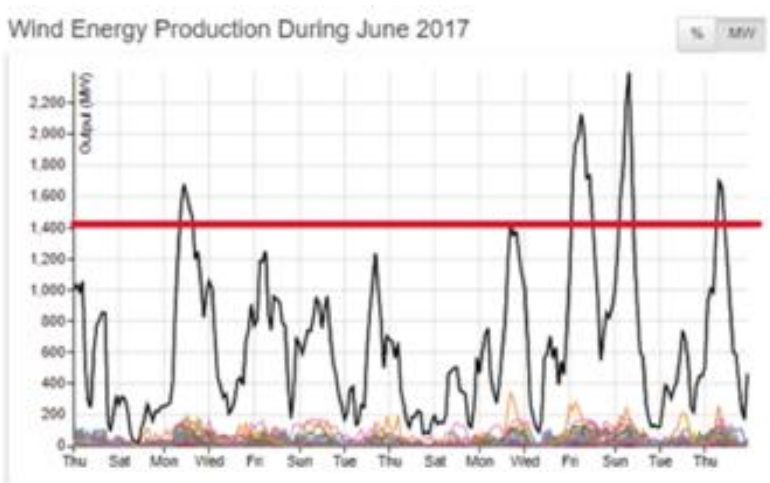

Fig. 1: Uncorrelated Wind power \& System Demand

This uncertainty in the availability of the power makes the generator an unsuitable candidate and for the grid operator it will be a very challenging task to sustain the grid.

\section{Proposed Implementation Strategy of Integration}

Since the quantum of available energy is directly correlated with the wind forecast (wind velocity and direction), adoption of a smart forecast engine can reduce the uncertainty. The figure below explains the relation.

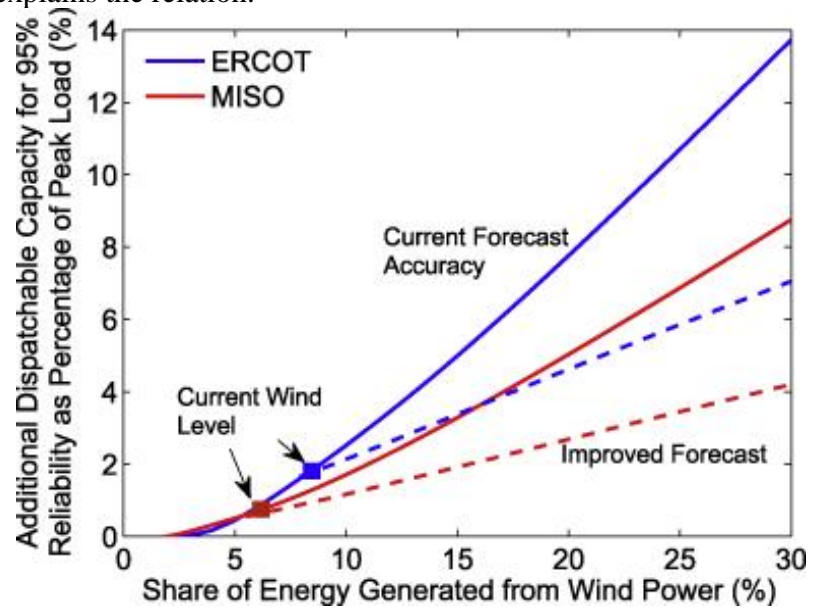

Fig. 2: Quantum of wind forecast is correlated with the quantum of predicted power

Several forecasting methods can be adopted, they are:

- Physical Method

- $\quad$ Statistical \& Fuzzy Neural Network method.

Further the forecast cab be for

- Short Term( several future hours)

- Medium Term (upto couple of Days).
Now propagation of gust of wind in different directions is accumulated to find out the total wind energy available in a specific period of time. The figure below explains the calculation method.
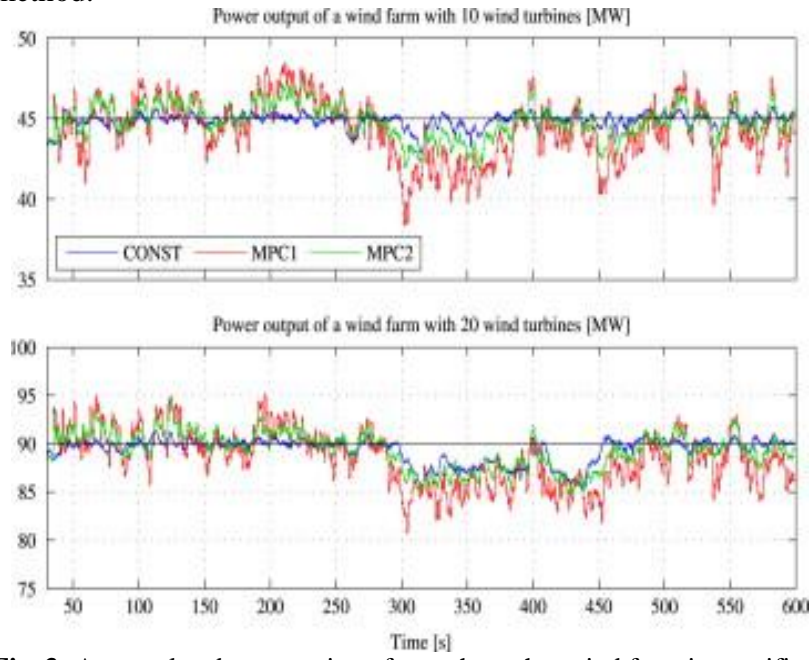

Fig. 3: Accumulated propagation of gust through a wind farm in specific time span

\section{A. Wind power Forecast for each Generator}

The energy output from a generator in a specific time span can be expressed as

$\int_{0}^{T} P t i=\mathrm{E}_{\mathrm{i}}$

Where,

$\mathrm{Pti}=$ Wind power available at a time $\mathrm{t}$ for generator $\mathrm{i}$.

$\mathrm{T}=$ Total Span of study

$\mathrm{Ei}=$ Total Quantum of renewable energy available in the total span for generator i.

Average power which can be fed to the grid by generator I for the same time span can be expressed as

$\mathrm{Ai}=\mathrm{Ei} / \mathrm{T}=$ mega watt.

Where,

$\mathrm{Ai}=$ Average Power from generator I for the above time span.

Figuring of is important to have a thought of the base MW, the generator may provide food when associated with the framework. Yet, in real condition, pretty much than a megawatt of energy will be accessible for infusion.

In the event that the breeze cultivate contains not very many generators, the decent variety in the yield will be high. Anyway, wind cultivates containing the extensive number of a generator; it will be conceivable to remunerate the pinnacle made by one generator by the made by another generator yield.

\section{B. Instantaneous Wind Power forecast for one wind Farm}

The total power output from the wind farm at any point of time can be expressed as

$\int_{i} P t i=\mathrm{W}_{\mathrm{i}}$

\section{where,}

$P$ ti $=$ Wind power available at time $\mathrm{t}$ for generator $\mathrm{i}$

$\mathrm{Wi}=$ Wind power available at time $\mathrm{t}$ from the wind farm.

If Wi does not have much variation, the farm would be ideal to integrate with grid. 


\section{Wind Power Forecast for one wind Farm}

The total energy output from wind farm in a specific time span can be expressed as;

$\iint_{0}^{T} P t i=\mathrm{W}$

\section{where,}

$\mathrm{W}=$ Total quantum of renewable energy available in the total span from the wind farm.

Similarity average power from the wind farm (Aw) for the same time span can be expressed as:

$\mathrm{Aw}=\mathrm{W} / \mathrm{T}$ megawatt.

With the assistance of authentic figure data and measurable examination, least power yield from the breeze cultivate are assessed. Leftover and difference of the real condition are processed against the assessed wind control figure. Remaining more than the client characterized resistance, can trigger a refined estimate.

Presently for a smooth and feasible task of the network, the tops in the power accessible bent should be cut and the valleys are to be filled as long as the generators are associated with the framework. A standout amongst the most well-known approaches to crest cutout or valley filling is to store the vitality. So amid the substantial breeze, power will be put away, and in the low breeze time frame, the put away vitality can be expended. Contingent upon the plausibility, area, vitality can be put away in the pump stockpiling plant or in a progression of batteries.

Quantum of capacity limit is guided by normal power from the breeze cultivate (Aw). In the event that the investigation comes about are predictable, is considered as the base MW for the breeze cultivate. The figure beneath clarifies the most extreme stockpiling limit required for a breeze cultivate.
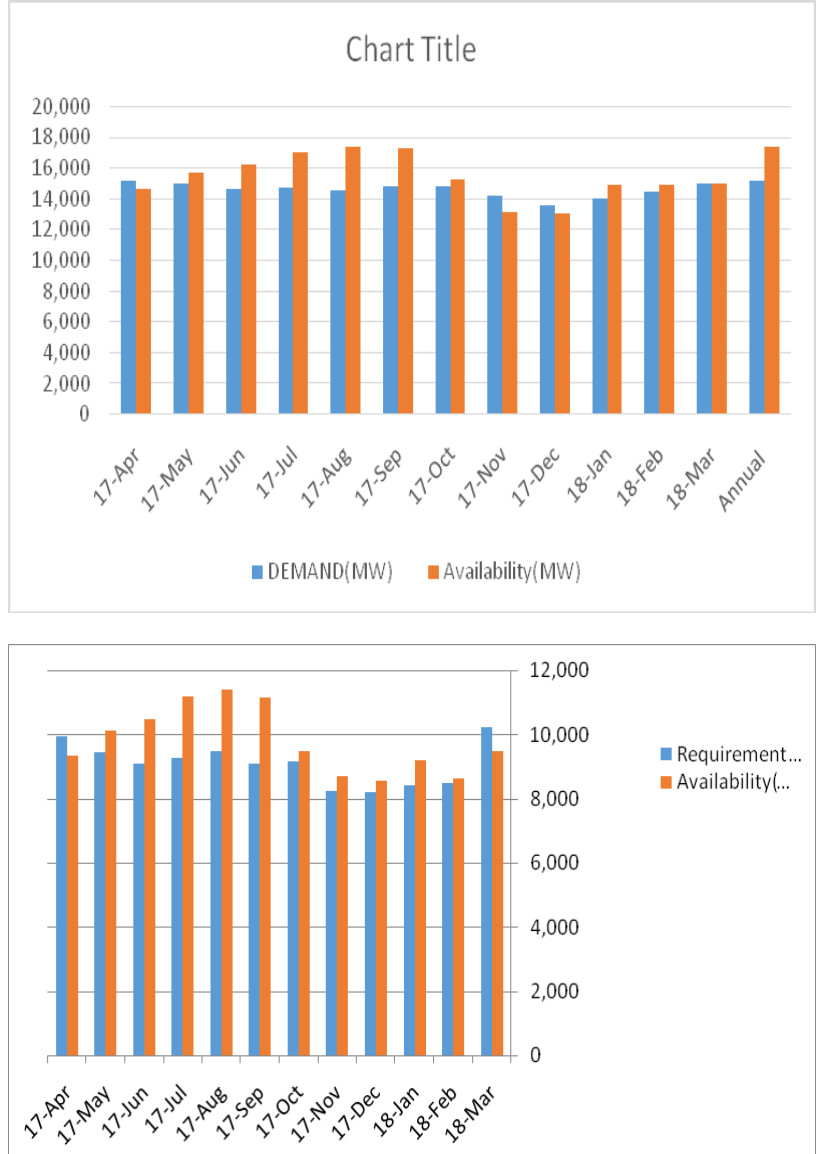

Fig. 4: Capacity determination of the storage to sustain different wind condition during operation
This is the way the physical and the operational difficulties are tended to. These activities are receptive activities took after by the event of the occasion. Given the multifaceted nature of regularly changing burden and climate conditions, a prescient examination took after by a proactive activity will settle the network in more intelligent way [4].

\section{Conclusion}

While comparing all renewable energy sources based on requirement, availability and surplus deficit Renewable energy is definitely the smart source of energy. Consider a range of specific variables that affect cost and performance and that might help explain the substantial difference in cost from one installation to another. The report further finds that both land-based and offshore wind costs continue to decrease managing it prudently contributes to the smart Grid.

\section{References}

[1] "India's Renewable Energy Sector -potential and Investment opportunities", Delhi International Renewable energy conference, 2010, 27-29 October 2010

[2] Eleventh Five Year plan (2007-2012) Agriculture, Rural Development, Industry, Services and Physical Infrastructure, Volume III, Planning Commission Government of India.

[3] Booklet no.9 'Solar Energy Centre', published by Ministry of Nonconventional energy sources, Government of India Table 1.

[4] Renewable plan for renewable energy integration.

[5] Government of India Central electricity authority report 2017.

[6] Wind Europe Annual statics, 2017.

[7] Evaluation of Wind Energy Potential as a Power Generation Source in Chad - Djamal Hissein Didane, Nurhayati Rosly, Mohd Fadhli Zulkafli, and Syariful Syafiq Shamsudin, International Journal of Rotating Machinery, Volume 2017, Article ID 3121875, 10 pages https://doi.org/10.1155/2017/3121875

[8] The Status and Future Prospects of Hydropower for Sustainable Water and Energy Development in Tanzania, Baraka Kichonge, Review Article (12 pages), Article ID 6570358, Volume 2018 (2018).

[9] National Renewable Energy Laboratory https://www.nrel.gov/docs/fy17osti/66861.pdf, Demonstrate Advancements in Wind Energy Research.

[10] Yan-Ting Lin, Pao-Hsiung Chiu, Chin-Cheng Huang, "An experimental and numerical investigation on the power performance of $150 \mathrm{~kW}$ horizontal axis wind turbine", Original research article Pages 85-93. 